\title{
Fungal Infection of Paranasal Sinus After COVID-19 Infection: A Tale of Two Fungi
}

\author{
Sunayana Mukesh Jangla ${ }^{1 *}$, Atul Shivaji Wadawkar ${ }^{2}$, Aniruddha Jayavant Randive ${ }^{2}$ and Sandip Sakharam Sakpal ${ }^{2}$ \\ 'Microbiology Section, Department of Pathology, Bhabha Atomic Research Centre Hospital, \\ Anushakti Nagar, Trombay, Mumbai 400-094, Maharashtra, India \\ ${ }^{2}$ Nucleus The Diagnostic Centre, "Rose” Flower Valley, Khadakpada Circle, Khadakpada, Kalyan (W), Maharashtra 421301, India
}

\begin{abstract}
Coronavirus disease (COVID-19) is an infection caused by Severe Acute Respiratory Syndrome Coronavirus-2 (SARS-CoV-2). Many twists and turns have occurred regarding its various aspects since the time it was first detected especially medical management. The COVID-19 symptom array has been ever expanding and recently secondary fungal infection has emerged as a top-up to the trouble. Post COVID-19 secondary fungal infections caused by Mucor have recently emerged as a cause of concern. However, infection caused by a combination of two fungal moulds from a site barring the lungs as a sequalae to the coronavirus disease comes as a surprise.
\end{abstract}

Keywords: Aspergillus, Covid-19, Immunocompromised, Infection, Mucor, Sinus,

\section{Introduction}

In December 2019, a cluster of cases of respiratory disease featuring pneumonia as the main symptom was reported in Wuhan city, Hubei province of People's Republic of China. This was later found to be caused by a novel coronavirus called Severe Acute Respiratory Syndrome Coronavirus-2 (SARS-CoV-2) and the disease caused by it as COVID-19. ${ }^{[1]}$ Just after SARS -CoV-2 has caused a riot in the human body and the patient is mending fences, post COVID-19 secondary infection comes into play.

In patients infected with COVID-19, an over-all state of immunosuppression occurs due to reasons like alteration of the innate immunity, caused by COVID-19 associated immune dysregulation featured by decreased $\mathrm{T}$ cells, including CD4 and CD8 cells. This encourages secondary bacterial and fungal infections especially in critically ill patients exposed to emergency invasive procedures, mechanical ventilation, prolonged hospital stays and lacunae in asepsis. Pre-existing fungal spores in the environment provide favourable conditions for fungal mould infections in such cases and there is a firm association observed between COVID-19 and increased fungal infections. ${ }^{[2,3]}$ Besides, fungi are found on most mucosal surfaces but the fungal microbiota is modified in disease states. ${ }^{[4]}$ Zygomycosis is an acute or chronic infection caused by fungi of the phylum Zygomycota seen in patients with altered immune system. It includes Order Mucorales and Entomopthorales.

Infection caused by fungi of the Order Mucorales is commonly called as Mucormycosis. Among Order
Mucorales, Rhizopus and Mucor are among the common causative agents causing the disease. ${ }^{[5]}$ Mucormycosis is an invasive fungal infection associated with high mortality and its incidence is rising in COVID-19 affected or recovered patients. Mucor has a low virulence potential and may be present in nasal mucosa of healthy individuals as a commensal. ${ }^{[2]}$ But in immunosuppressive states, it may invade paranasal sinuses and rarely spread to adjacent organs like eyes and brain. Invasive Pulmonary Aspergillosis (IPA) in such patients is a known concern. [6] However, reports of infection of the sinus caused by Aspergillus or a combination of two moulds are sparse. We hereby, report one such case involving right maxillary sinus in a patient days after he was infected with COVID-19. It was clinically diagnosed as Mucormycosis of the sinus. However, microbiological diagnosis was an eye-opener as it showed the infection to be caused by Mucor species along with Aspergillus flavus.

\section{Case Report}

38 years old male presented with swelling, fullness, numbness and pain over right-sided cheek for seven days. There was no fever, headache or eye related symptoms. He did not have any co-morbidities nor major illness in the past. He was detected as a confirmed case of COVID-19 infection one month back for which he was hospitalised for two weeks. During this course, he was given intravenous methylprednisolone 40 milligram twice a day for ten days.

On examination, there was swelling and tenderness over right cheek but no overlying redness or discharge from affected area. Sino-nasal endoscopy showed pale mucosa 
with black areas of tissue necrosis. On current visit, his fasting blood sugar was $122 \mathrm{mg} / \mathrm{dl}$ and $\mathrm{HbAlc}$ was $8.7 \%$. Bleeding time and Clotting time and prothrombin test were normal. No other investigations were done. Gadolium-enhanced Magnetic Resonance Imaging (MRI) of paranasal sinuses showed thickened mucosa with partial opacification of right sided maxillary sinus [Fig.1].

Blood sugar levels were controlled using insulin drip and surgical debridement of the involved area was done. Tissue was sent for $10 \%$ Potassium hydroxide $(\mathrm{KOH})$ mount, fungal culture and histopathological diagnosis. Intravenous liposomal Amphotericin-b was given in a dose of 350 milligram for seven days after which the patient was discharged. On gross examination, the tissue was red with intermittent black material [Fig.2]. $10 \% \mathrm{KOH}$ mount showed branching septate and aseptate hyphae. Fungal culture was done on Sabouraud Dextrose Agar (SDA) without and with antibiotics (chloramphenicol and gentamicin) in duplicate. One set was incubated at $37{ }^{\circ} \mathrm{C}$ and other at $24{ }^{\circ} \mathrm{C}$ respectively. At the end of 24 hours there was growth on all plates and it was green velvety along with slight grey coloured growth over it. Lactophenol Cotton Blue (LPCB) mount from the growth showed septate branching hyphae with long conidiophores and phialides covering entire vesicle pointing out in all directions and few broad aseptate hyphae without rhizoids and without distinct columella. After 48 hours growth increased in size and became black, dense, woolly. [Fig.3]. Lactophenol Cotton Blue (LPCB) mount from the growth

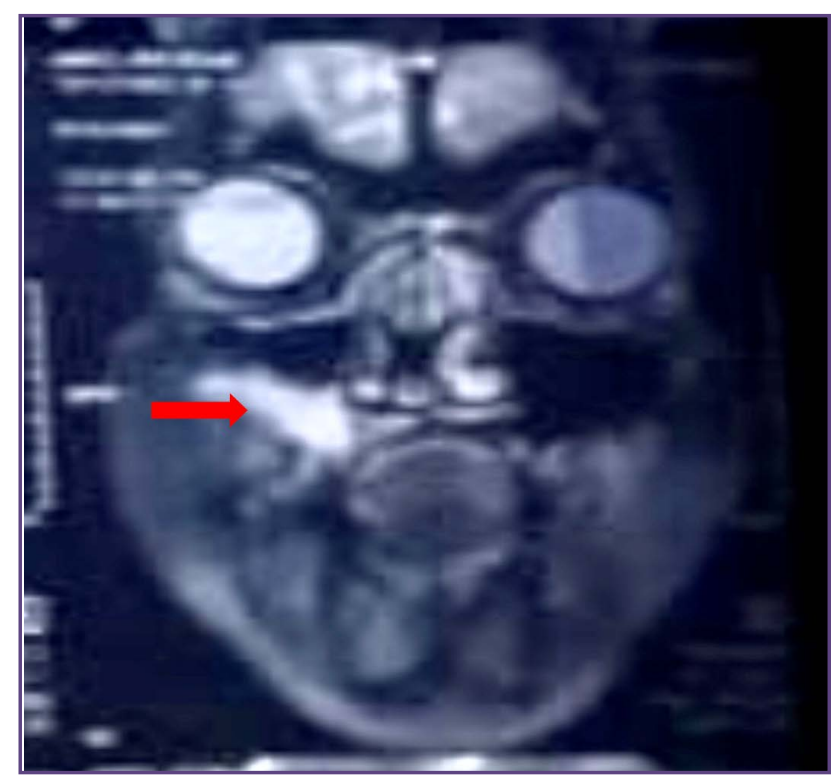

Fig. 1: MRI with contrast showing partial opacification of right maxillary sinus. showed the same findings as above [Fig.4]. Slide culture from the growth also showed same findings.

Hence, it was reported as right sided maxillary sinus infection caused by Mucor species and Aspergillus flavus. Histopathology of the tissue was morphologically consistent with Mucor species and Aspergillus species [Fig.5]. On discharge, he was put on insulin with strict blood sugar level monitoring. Patient was called for followup after a week and there-after every fifteen days up-till three months. There was no recurrence or spread seen and the patient recovered.

\section{Discussion}

In COVID-19 patients, there is severe injury to immune response due to reasons like decrease in lymphocyte count and T-cell subgroup causing immune dysregulation. COVID-19 can cause extensive pulmonary disease and subsequent alveolo-interstitial pathology which predisposes to various fungal infections of airways including sinuses and lungs ${ }^{[2,7]}$ Hence, it is a state of immunosuppression. ${ }^{[2]}$ The drugs that are a part of the COVID-19 treatment strategy are antiviral, antibiotics and immune-boosters while the role of glucocorticoids as anti-inflammatory drug was controversial till late as they have many side effects including steroid induced hyperglycaemia. ${ }^{[8,9]}$ But low dose, short duration dexamethasone can be beneficial in moderate to severe illness as it can alleviate the early inflammatory response to coronavirus infection and help in viral clearance and has less side effects. ${ }^{[8,9]}$

Our patient was given steroids while he was hospitalised for COVID-19. Though, he was not a known case of

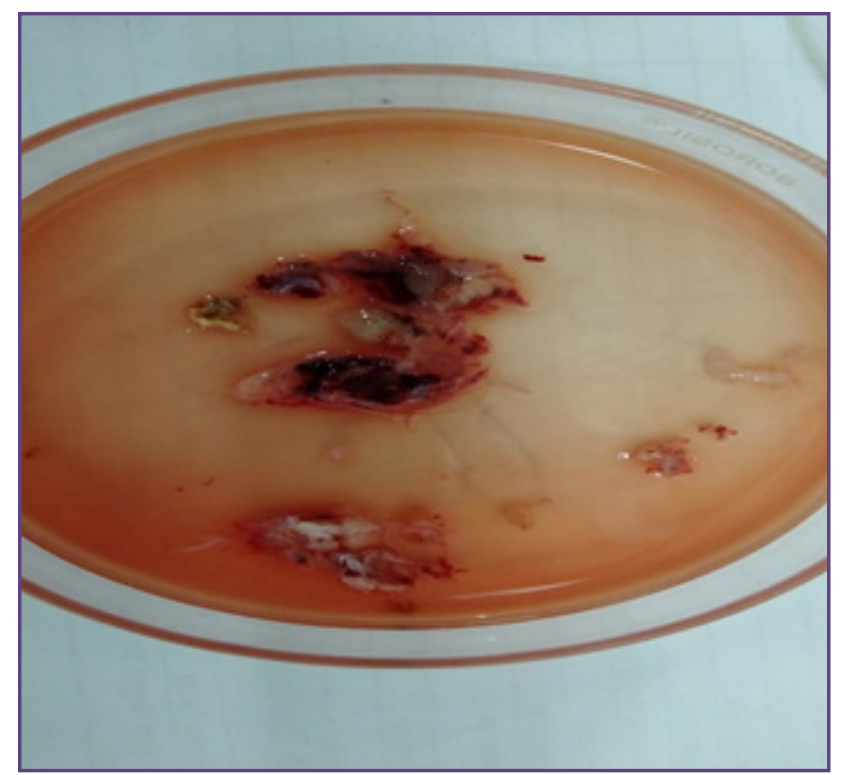

Fig. 2: Tissue Sample of the patient. 


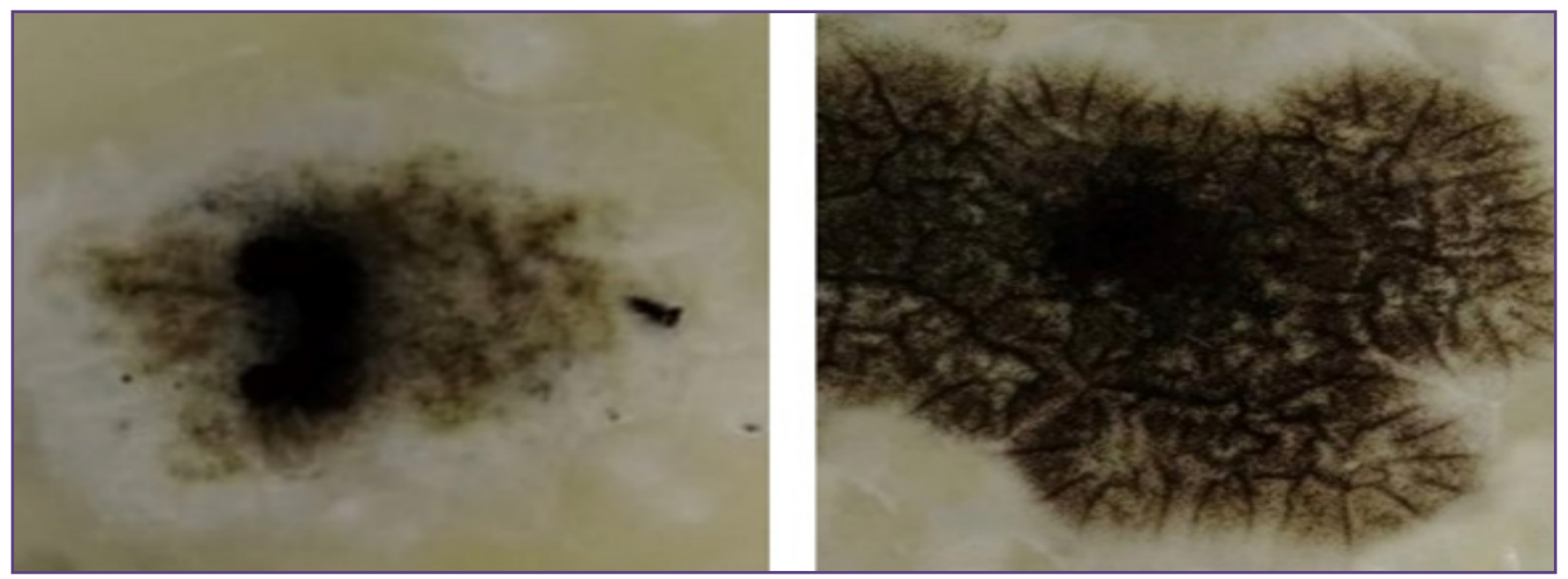

Fig. 3: Growth on SDA at 24 hours and 48 hours.

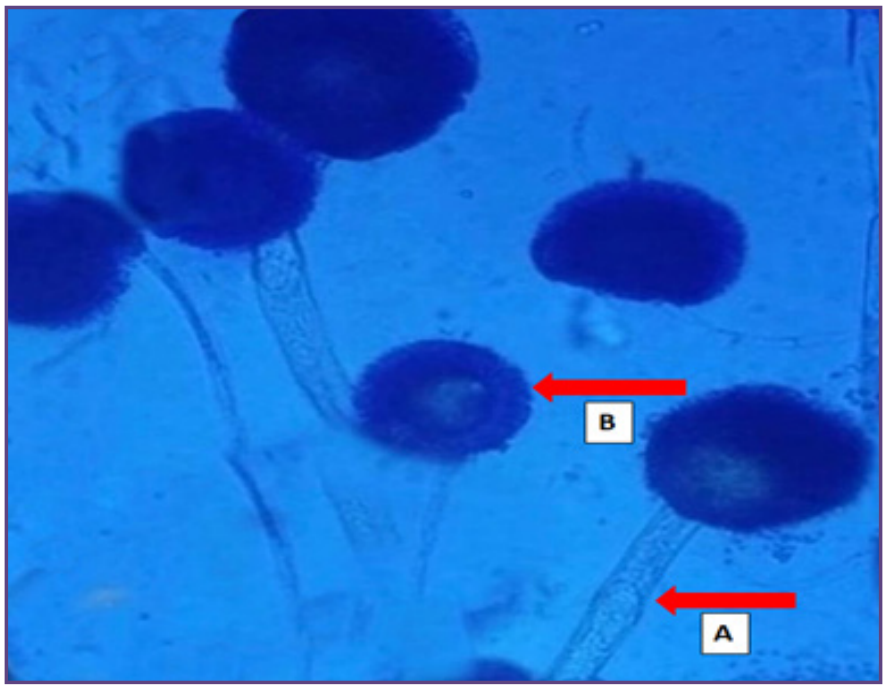

Fig. 4: LPCB mount from growth showing Mucor (A) and A. flavus (B).

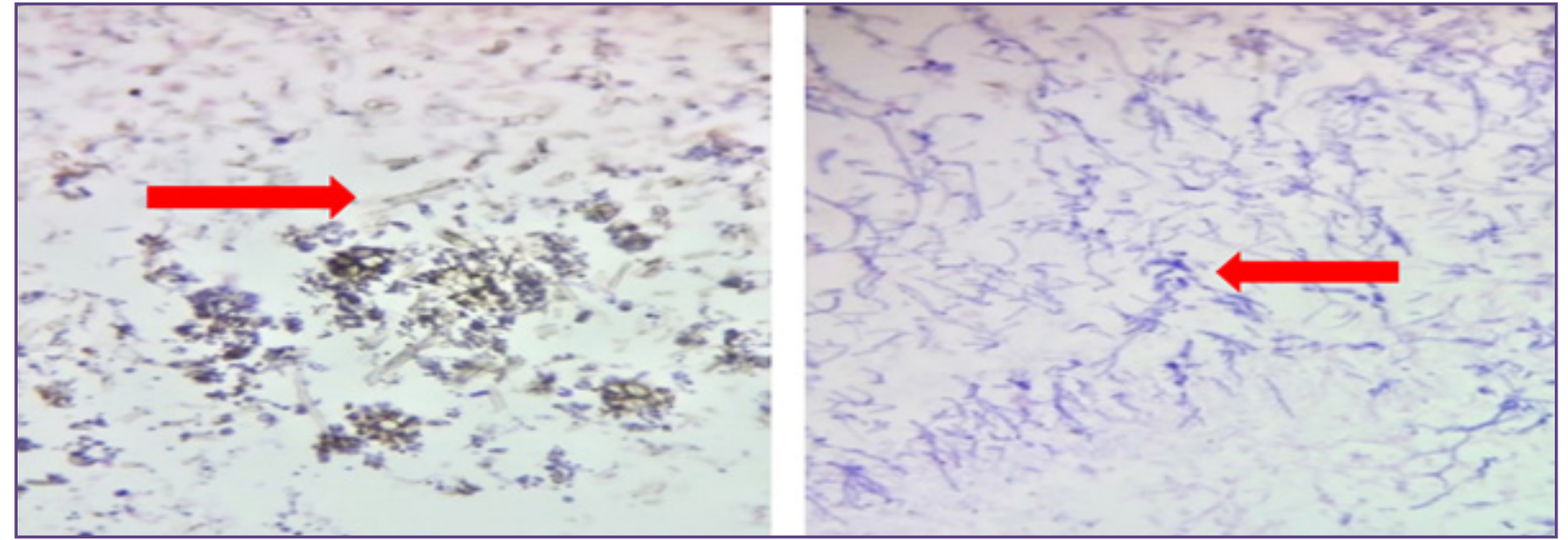

Fig. 5: Histopathology of sample showing broad hyphae of Mucor and septate hyphae of Aspergillus (H \&E, x40). 
diabetes mellitus his glycosylated haemoglobin and fasting blood sugar were high at the time of his presentation. This could be steroid induced hyperglycaemia which is a known side effect of this drug that was given to him for his COVID infection or he may have been detected as having diabetes mellitus then. Invasive fungal sinusitis is an uncommon disease commonly caused by hyaline moulds like Aspergillus species including A. flavus, Mucor and Fusarium in immunocompromised patients with a high mortality. ${ }^{[10]}$ Invasive mould infection commonly occurs when a large burden of spores which are commonly present in the environment is deposited on mucosal membranes without an effective phagocytic host defence. $^{[11]}$

In states of altered immune system, Zygomycosis or Mucormycosis occurs ${ }^{[2]}$ There is an outright risk of Mucormycosis in diabetic COVID-19 patient receiving steroids which were also the characteristics of our patient. It is commonly caused by Rhizopus followed by Mucor. ${ }^{[5]}$ Clinical trademark of this infection is tissue necrosis seen as black discharge, eschar or ulcer on examination. [9] The sample received from our patient from right maxillary sinus too had similar appearance. Ethmoidal and maxillary sinuses are commonly involved. ${ }^{[2]}$ In our case too, maxillary sinus was involved. Generally, symptoms of mucor develop 3-42 days after development of COVID-19. ${ }^{[12]}$

Our patient presented approximately seven to ten days after discharge from hospital. The mould with aseptate hyphae was identified as Mucor as it had no rhizoids nor a distinct columella. Aspergillus came as a dismay in this case as paranasal sinus infection in post-COVID-19 patients has been recently reported to be caused by Mucor ${ }^{[2,9]}$ Thus, the deadly duo of steroid and hyperglycaemia adding to the COVID-19 induced immunosuppression state and ubiquitously present fungal spores predisposed him to a combined infection of Aspergillus and Mucor.

The infection was indicated by $\mathrm{KOH}$ mount, proven on culture and confirmed by histopathology. Treatment consists of surgical debridement, antifungals, correction of underlying risk factor and adjuvant therapy. Surgical debridement should be done at the earliest. Amphotericin-b remains the drug of choice against Mucorales and many Aspergillus species. ${ }^{[13]}$ Its liposomal preparation is preferred to reduce toxicity. Our patient was also managed in a similar way along with aggressive control and monitoring of blood sugar levels. Fortunately, the involvement of sinus was not extensive and there was no spread to adjacent organs. Hence prognosis was better.

\section{Conclusion}

Both the erstwhile fungi, Mucor and Aspergillus are omnipresent. Recently. cases of fungal infections of paranasal sinuses by Mucor have been reported. But our case is an unexpected post coronavirus disease doom and an addition to the lessons learnt about the new and longterm manifestations of the COVID-19 infection. In such patients especially those with additional risk factors, not only Mucor but the duo of Mucor and Aspergillus is worrisome and a high index of suspicion helped us clinch this diagnosis.

\section{Acknowledgement}

None

\section{Funding}

None

\section{Competing Interests}

None

Declaration of patient consent: The authors certify that they have obtained all appropriate patient consent forms. In the form patient has given his consent for the images and other clinical information to be reported to the journal. The patient understands that his name and identity will not be published and due efforts will be made to conceal the identity of the patient.

\section{References}

1. Dikid T, Chaudhary S, Goel K, Padda P, Sahu R. Responding to COVID-19 pandemic: Why a strong health system is required. Indian J Med Res 2020; 151:140-5

2. Sharna S, Grover M, Bhargava S, Samdani S, Kataria T. Post coronavirus disease mucormycosis: a deadly addition to the pandemic spectrum. J Laryngol Otol 2021;1-6.

3. Dallalzadeh O L, Ozzello J D, Liu Y C, Kikkawa O D, Korn S B. Secondary infection with rhino-orbital cerebral mucormycosis associated with COVID-19. Orbit 2021;23;14

4. Limon J J, Skalski H J, Underhill M D, Commensal fungi in Health and Disease. Cell Host Microbe 2017;9;22(2):156165

5. Chander J. A Textbook of Mycology 2nd New Delhi : Mehta Publishers ;2002:p. 286-299

6. Lai Chih-Cheng, Yu Weng-liang.COVID-19 associated with pulmonary aspergillosis: A literature review.J Microbiol Immunol Infect $2021 \mathrm{Feb}$;54(1):46-53.

7. Hai-Yan Fu, Yu Luo, Jian-Peng Gao, Lin Wang, Hong-Juan Li, Xiang Li, Lian Xue et al. Effects of Short-Term Low-Dose Glucocorticoids for Patients with Mild COVID-19. BioMed Res Intl, vol. 2020, Article ID 2854186,

8. Sarkar S, Gokhale T, Choudhary SS, Deb AK. COVID-19 and orbital mucormycosis. Indian J Opthalmol 2021;69:1002-4. 
9. Soman R, Sunavala A. Post COVID-19 Mucormycosis -from the Frying Pan into the Fire. Macleods 2021;69

10. Hamid M, Ahmed Sitt, Jamii Abdulmoneim, Al-Qahatani Ali. Allergic Fungal Rhinosinusistis Caused by Aspergillus flavus. Annals of African Surgery 2019 Aug;16(1):75-77.

11. Chen Chien-Yuan, Sheng Wang-Huei, Cheng Aristine, Chen Yee-Chun, Tsay Woei, Tang Jih-Luh et al. Invasive fungal sinusitis in patients with hematological malignancy: 15 years experience in a single university hospital in Taiwan. BMC Infect Dis 2011 Sep; 11:250

12. Sen M, Lahane S, Lahane P T,Parekh R,Honavar G S.Mucor in a Viral Land: A Tale of Two Pathogens. Indian J Opthalmol 2021;69(2):255-252

13. Singh V. Fungal rhinosinusitis: Unravelling the disease spectrum. J Maxillofac Oral Surg 2019; 18(2):164-179

*Corresponding author:

Dr. Sunayana M Jangla, Room no.6, Microbiology section, Bhabha Atomic Research Centre Hospital (BARCH), Anushakti Nagar, Trombay, Mumbai 400-094, Maharashtra, India

Phone: +91 9819039287

Email: sunayanajangla79@gmail.com

Date of Submission $\quad: 17 / 06 / 2021$

Date of Final Revision : 10/08/2021

Date of Acceptance $\quad: \mathbf{1 0} / 08 / 2021$

Financial or other Competing Interests: None.

Date of Publication : $\quad$ 30/07/2021 